\title{
BMJ Open Comparison between urban and rural mortality in patients with acute myocardial infarction: a nationwide longitudinal cohort study in South Korea
}

\author{
Hye Sim Kim (D) , ${ }^{1}$ Dae Ryong Kang, ${ }^{2}$ Inah Kim (D) , ${ }^{3}$ Kyungsuk Lee, ${ }^{4}$ Hoon Jo, ${ }^{5}$ \\ Sang Baek Koh ${ }^{5}$
}

To cite: Kim HS, Kang DR, Kim I, et al. Comparison between urban and rural mortality in patients with acute myocardial infarction: a nationwide longitudinal cohort study in South Korea. BMJ Open 2020;10:e035501. doi:10.1136/ bmjopen-2019-035501

- Prepublication history for this paper is available online. To view these files, please visit the journal online (http://dx.doi. org/10.1136/bmjopen-2019035501).

Received 04 November 2019 Revised 18 February 2020 Accepted 19 February 2020
Check for updates

(C) Author(s) (or their employer(s)) 2020. Re-use permitted under CC BY-NC. No commercial re-use. See rights and permissions. Published by BMJ.

For numbered affiliations see end of article.

Correspondence to Professor Sang Baek Koh; kohhj@yonsei.ac.kr

\section{ABSTRACT}

Objectives This study investigated the risk associated with interhospital transfer of patients with acute myocardial infarction (AMI) and clinical outcomes according to the location of the patient' residence.

Design A nationwide longitudinal cohort.

Setting National Health Insurance Service database of South Korea.

Participants This study included 69899 patients with AMI who visited an emergency centre from 2013 to 2015, as per the Korea National Health Insurance Service database.

Primary outcome measure The clinical outcome of a patient with AMI was defined as mortality within 7 days, 30 days and 1 year.

Results Clinical outcomes were analysed and compared with respect to the location of the patient's residence and occurrence of interhospital transfer. We concluded that the HR of mortality within 7 days was 1.49 times higher $(95 \%$ $\mathrm{Cl} 1.18$ to 1.87) in rural patients than in urban patients not subjected to interhospital transfer and 1.90 times higher (95\% Cl 1.13 to 3.19$)$ in transferred rural patients than in non-transferred urban patients.

Conclusions To reduce health inequality in rural areas, a healthcare policy considering regional characteristics, rather than a central government-led, catch-all approach to healthcare policy, must be formulated. Additionally, a local medical emergency delivery system, based on allocation of roles between different medical facilities in the region, must be established.

\section{INTRODUCTION}

Acute myocardial infarction (AMI), one of the three major medical emergencies including stroke and major trauma, is a medical condition with high incidence and fatality rates; however, early mortality and disability associated with AMI can be greatly reduced if adequate and timely medical care is provided. ${ }^{1}$ The transfer of a patient to another hospital or specialist to obtain appropriate medical treatment in a timely
Strengths and limitations of this study

- The strength of this study is that all patients who visited the emergency room for acute myocardial infarction were analysed using the National health claims database in Korea.

- Using a nationwide longitudinal database enabled the identification of medical use of the overall population, so these results can be generalised to the population in South Korea.

- Due to the nature of the health insurance claim data, reflecting on the severity of disease and patients' clinical characteristics during visits was not possible.

manner may affect the clinical outcomes of the patient. ${ }^{2-5}$ Moreover, many patients with emergency conditions who visit emergency departments are often transferred to other medical centres due to limited capacity, including shortage of medical facilities and professionals. This, in turn, causes delayed arrivals of emergency patients at the final care facilities and, thus, has an effect on patient clinical outcomes. ${ }^{67}$

Most medical centres that can offer specialised care are concentrated in urban areas with higher population densities and higher incomes, and correspondingly, rural areas experience shortages of specialised care facilities and personnel, which hinders the provision of appropriate and timely emergency medical care. ${ }^{8-10}$ The Emergency Medical Service Act in Korea divides emergency medical institutions into regional emergency medical centre, local emergency medical centre and local emergency medical facilities. In terms of medical resources, the emergency medical centre is a hospital equipped with more specialised facilities and human 
resources than is an emergency medical facility. In terms of treatment capacity, regional emergency medical centres can provide more advanced emergency medical services to treat more severe emergency patients than can local emergency medical centres. In fact, while there are 15 regional emergency medical centres in urban areas, there are none in rural areas, and there are 136 local emergency medical institutions in urban areas and 72 in rural areas. In such conditions, rural healthcare authorities are forced to undertake extensive interhospital transfers of emergency patients, ${ }^{11}$ which ultimately leads to health inequalities between the rural and urban communities.

According to previous studies, the implementation rates of medical interventions and emergency care described in the guidelines for the management of patients with AMI was reported to be lower in rural than in urban areas, ${ }^{12-15}$ and mortality within 30 days for emergency patients was reported to be higher in rural than in urban areas. ${ }^{10} 15$ Furthermore, with respect to deaths that could have been avoided if appropriate and timely healthcare had been provided, the mortality rate was reported to be higher in rural areas than in urban areas, indicating that rural communities suffer from health disparities with regard to many diseases. ${ }^{16}$

Other countries continuously conduct research on rural health issues with respect to medical emergencies, ${ }^{8} 10^{13-15}$ along with diverse research on the effects of interhospital transfers. ${ }^{589}$ In contrast, most research conducted in South Korea has focused on the status of particular diseases and healthcare systems in rural areas only, and research on interhospital transfers has also been limited to one individual hospital. This has resulted in insufficient research on the rural-urban comparison of interhospital transfer of patients with AMI and their clinical outcomes.

Therefore, this study examines the OR of occurrence of interhospital transfer in patients with AMI who visited an emergency department, using the National Health Insurance claims data, and then compares the HR of mortality between rural and urban areas; it aims at identifying the negative effects of such transfers and provide a basis for policy-making towards achieving enhanced accessibility of emergency medical care and to ensure health equality between rural and urban communities.

\section{METHODS}

\section{Database and participants}

This was a nationwide longitudinal cohort study using data from the national health claims database. In South Korea, approximately $98 \%$ of the population is included in the national health insurance system. Thus, claims data are representative of the whole population treated for a specific illness.

This study included 69899 patients diagnosed with AMI during their emergency centre visits, from January 2013 to December 2015, who were identified using medical claims data from the National Health Insurance Service.
A patient with AMI is defined as a person diagnosed with code I21 based on the International Classification of Diseases, 10th revision disease classification.

\section{Variables}

The cities were divided on the basis of the administrative districts of residence of patients. The administrative districts of Korea were classified as capital city, metropolitan cities and provinces. Also, each municipality was divided into districts, cities and counties as lower administrative districts in consideration of financial independence, city type and population size. According to the division of administrative districts, the cities were classified as follows: the cities of administrative districts in the capital city and metropolitan areas were classified as urban (with approximately half a million residents); the cities of administrative districts in the provinces were classified as suburban (with fewer than 50 000-5 00000 residents) and the counties of administrative districts in the provinces were classified as rural (fewer than 50000 residents). ${ }^{17}$ Based on these standards, social and economic support is differentially applied. In this study, the same definition was applied to classify cities.

The results of emergency medical interventions in the claims database are categorised into interhospital transfer, discharge, death, return and continuance (scheduled outpatient). Return is also included in interhospital transfers. However, in the claim data, return is defined as the case where it is transferred to a nursing institution after appropriate treatment. Therefore, in this study, the interhospital transfer was defined as a transfer within 1 day of visiting the emergency room, and return was not included in interhospital transfer. This is to ensure that the return patient, transferred to a nursing institution after proper treatment, is not included as a patient in the interhospital transfer category.

The clinical outcome of patients with AMI confirmed death within 7 days, 30 days and 1 year after the interhospital transfer through the death database of the claims database.

\section{Statistical methods}

To compare the characteristics of study subjects according to the area of residence, $\chi^{2}$ and analysis of variance (ANOVA) tests were performed for categorical variables and for continuous variables, respectively. Scheffe's posthoc test was also performed to test the differences between groups after ANOVA confirmed statistical significance. Moreover, a multivariable logistic regression was performed to obtain the OR of interhospital transfers with respect to classification of the area of residence.

As concerns the clinical outcomes of patients with AMI, a Cox proportional hazard model was used to determine the HR of mortality within 7 days, 30 days and 1 year. The age stratification analysis was conducted because the age of a patient can have a significant effect on clinical outcomes. In Korea, the definition of the elderly (senior) varies depending on the law. The national basic 
Table 1 General characteristics according to the area of residence of patients with acute myocardial infarction

\begin{tabular}{|c|c|c|c|c|}
\hline Characteristic & Urban $^{a}(n=28688)$ & Suburban $^{b}(n=31559)$ & Rural $^{c}(n=9652)$ & $P$ value \\
\hline Age (years) & $64.09 \pm 13.44$ & $64.27 \pm 13.82$ & $68.80 \pm 12.94$ & $\begin{array}{l}<0.001 \\
a, b<c\end{array}$ \\
\hline National Health Insurance (KRW) & $106995 \pm 112690$ & $104292 \pm 99766$ & $97539 \pm 96822$ & $\begin{array}{l}<0.001 \\
c<b<a\end{array}$ \\
\hline Insurance categories & & & & $<0.001$ \\
\hline Public employee insurees & $1782(7.15)$ & $2305(8.47)$ & $903(11.28)$ & \\
\hline Employed insurees & $13155(52.78)$ & $14174(52.08)$ & $3619(45.2)$ & \\
\hline Medical aid & $2009(8.06)$ & $1786(6.56)$ & $660(8.24)$ & \\
\hline Regional insurees (Rural) & $246(0.99)$ & $533(1.96)$ & $2726(34.05)$ & \\
\hline Charlson's comorbidities index & & & & $<0.001$ \\
\hline $3+$ & $12436(43.35)$ & $14073(44.59)$ & $5116(53.00)$ & \\
\hline Transfer, yes & $1044(3.64)$ & $1883(5.97)$ & $1054(10.92)$ & $<0.001$ \\
\hline
\end{tabular}

Data are presented as means \pm SD or numbers and percentages. $\mathrm{P}<0.05$ was accepted as statistically significant.

living security act defines the elderly based on the age of 65 years, but the act prohibiting age discrimination in employment and elderly employment promotion is based on the age of 55 years. Therefore, in this study, stratification analysis was divided at 55 years old to exclude the age effects of the elderly population.

Statistical analysis was performed using SAS 9.4 (SAS., Cary, North Carolina, USA) and R 3.5 (Institute for Statistics and Mathematics, Vienna, Austria; www.R-project. org).

\section{Patient and public involvement}

We used administrative claim data and did not involve patients in this study.

\section{RESULTS}

\section{General characteristics}

The average age of the study subjects in rural areas was $68.80 \pm 12.94$, which was higher than that of patients in urban and suburban areas, and the percentage of patients who underwent interhospital transfer in rural areas was
$10.92 \%$, which was three times higher than that in urban areas and 1.83 times higher than that in suburban areas, with the differences found to be statistically significant (table 1).

\section{Risk of interhospital transfer for patients with AMI with respect to area of residence}

The OR for interhospital transfer with respect to the area of residence was examined, and the crude OR for interhospital transfer in suburban areas was 1.68 times higher (95\% CI 1.56 to 1.82 ) than that in urban areas, and in rural areas was 3.25 times higher (95\% CI 2.97 to 3.55 ) than that in urban areas. Adjustment for sex, age and comorbidities virtually did not change the estimated ORs (table 2).

\section{Clinical outcomes of patients with AMI}

The HRs of mortality in patients with AMI within 7 days, 30 days and 1 year after emergency centre visit were investigated using an age-stratified analysis. The results revealed that for the patients with AMI aged 55 or younger, the HRs of mortality within 7 days and 30 days were 1.51

Table 2 Predicting factors of interhospital transfer of patient with acute myocardial infarction

\begin{tabular}{|c|c|c|c|c|}
\hline \multirow[b]{2}{*}{ Characteristic } & \multicolumn{4}{|c|}{ OR $(95 \% \mathrm{Cl})$} \\
\hline & Crude & Model 1 & Model 2 & Model 3 \\
\hline Urban & ref. & ref. & ref. & ref. \\
\hline Rural & 3.25 (2.97 to 3.55$)$ & 3.19 (2.92 to 3.49 ) & 3.20 (2.93 to 3.50$)$ & 3.19 (2.92 to 3.49$)$ \\
\hline
\end{tabular}

Model 1: Crude+sex.

Model 2: Crude+sex+age.

Model 3: Crude+sex+age+Charlson's comorbidities index. 
Table 3 HRs of mortality within 7, 30 day and 1 year of rural compared with urban patients with acute myocardial infarction

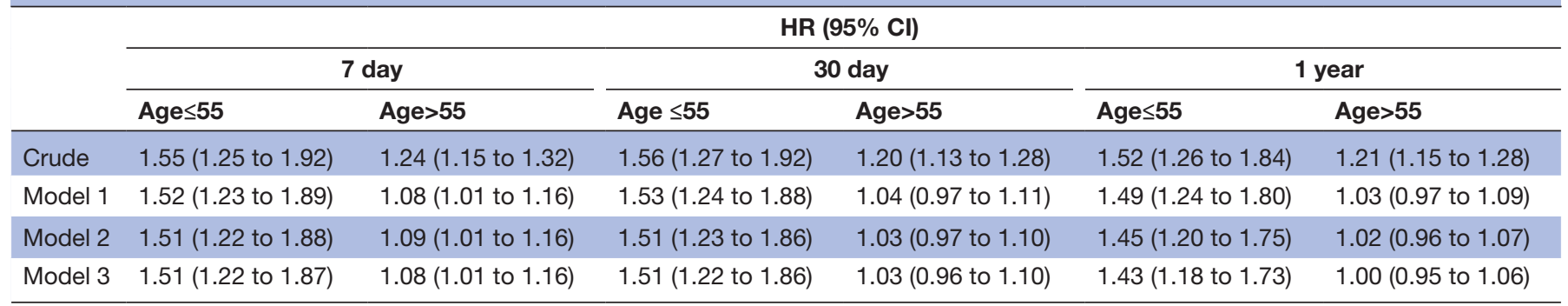

Model 1: age, sex.

Model 2: age, sex, transfer.

Model 3: age, sex, transfer, Charlson's comorbidities index.

times higher in rural than in urban areas, and the HR of mortality within 1 year was also higher by 1.43 times $(95 \%$ CI 1.18 to 1.73 ) in rural areas. For patients with AMI aged 55 or older, the HR of mortality within 7 days was 1.08 times higher (95\% CI 1.01 to 1.16 ) in rural than in urban areas, which was statistically significant (table 3 ).

\section{Clinical outcomes for patients with AMI with respect to the area of residence and interhospital transfer}

The clinical outcomes of patients with AMI aged 55 or younger were examined with respect to area of residence and interhospital transfer, and the results revealed that the HR of mortality within 7 days was 1.49 times higher (95\% CI 1.18 to 1.87 ) and the HRs of mortality within 30 days and 1 year were 1.51 times higher (95\% CI 1.21 to 1.88 ) and 1.42 times higher (95\% CI 1.16 to 1.74 ), respectively, in rural than in urban patients without interhospital transfer. Furthermore, if rural patients with AMI were subjected to transfer, their HRs of mortality within 7 days and 1 year were 1.90 times higher (95\% CI 1.13 to 3.19 ) and 2.23 times higher (95\% CI 1.47 to 3.39), respectively, than those of urban patients (figure 1).

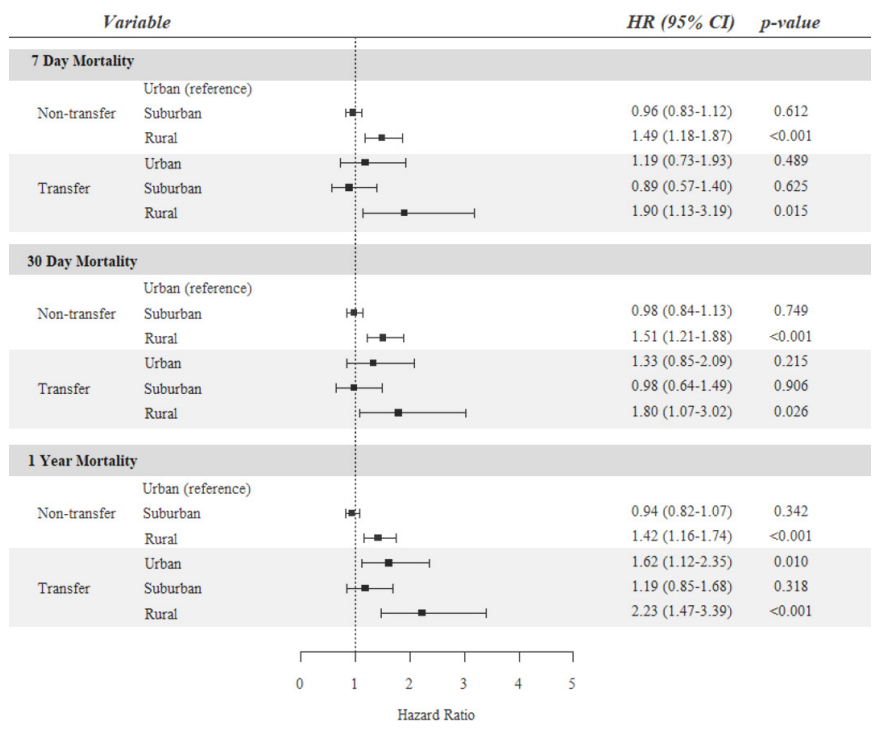

Figure 1 HRs of mortality with respect to interhospital transfer and area of residence in patients with AMI (adjusted for age, sex, Charlson's comorbidities index). AMI, acute myocardial infarction.

\section{DISCUSSION}

Interhospital transfer is an essential component of emergency care, a process required for more specialised medical care ${ }^{18}$ and, consequently, could be deemed an important step for ensuring continuity of emergency care as well as patient safety. However, interhospital transfer itself may act as a risk factor with respect to the clinical outcomes of patients with AMI, who require timely and appropriate care, as it often causes delayed arrival of the transferred patient at a hospital capable of providing the necessary care. ${ }^{819}$ The frequency of interhospital transfer may vary in different areas, especially between rural areas suffering shortages of medical resources and urban areas, where such resources are concentrated, thereby causing differences in the resulting clinical outcomes. This study was conducted to identify such differences.

Interhospital transfer rates with respect to location of patient residence were examined using National Health Insurance claims data, and the results revealed that such transfers were most frequent in rural areas: the OR for interhospital transfer was found to be 3.19 times higher (95\% CI 2.92 to 3.49 ) in rural than in urban areas. This is consistent with findings of many other studies reporting a high rate of transfer in non-urban areas. ${ }^{720} 21$

Age-stratified analysis was performed to take into account old-age mortality when examining the clinical outcomes of patients with AMI because of a tendency towards higher concentrations of elderly patients in rural areas. This revealed that rural patients aged 55 or younger had a nearly 1.5 times higher HR of mortality within 7 days, 30 days and 1 year compared with urban patients, while patients aged 55 or older in rural areas had a 1.08 times (95\% CI 1.01 to 1.16) higher HR of mortality within 7 days compared with those in urban areas. With respect to the area of residence, the clinical outcomes of patients with AMI were found to be less favourable in rural than in urban areas, and rural patients in particular, even non-elderly, experienced less favourable clinical outcomes. This might be caused by delays in arrival times at appropriate medical centres for the transferred patients because of poor access to healthcare services in rural areas. ${ }^{1015}$ Furthermore, the shortages of healthcare facilities and personnel capable of providing 
acceptable care in rural areas hinders the recommended early diagnosis and treatment, ${ }^{12-15}{ }^{22}$ causing disparities in the quality of healthcare services between rural and urban areas, thereby having a negative effect on the clinical outcomes of patients with AMI in desperate need of prompt treatment.

Moreover, the clinical outcomes of patients with AMI aged under 55 years with respect to the area of residence and interhospital transfer were examined to determine the effects of such transfers on patient outcomes. The results revealed that patients with AMI not subjected to interhospital transfer in rural areas had higher HRs of mortality within 7 days, 30 days and 1 year than urban patients, ranging from 1.42 to 1.51 times. If transferred, rural patients with AMI had 1.90 times higher (95\% CI 1.13 to 3.19 ) HR of mortality within 7 days than nontransferred urban patients. The clinical outcomes of rural patients with AMI, even without undergoing interhospital transfer, were less favourable than those of urban patients. For rural patients with AMI undergoing transfer, the HR increased further. This is consistent with the findings of Bhuyan $e t a l$, who reported that rural patients with AMI had less favourable clinical outcomes than urban patients and is also consistent with the findings of many previous studies reporting that transferred patients had higher risks of complications and mortality than non-transferred patients. ${ }^{392324}$ Moreover, interhospital transfer, even after adjustment for general characteristics, disease severity, and treatment time of patients, has been identified as a predictive factor that increases mortality. ${ }^{15}$ Therefore, the initial emergency care visit should be arranged to ensure the availability and accessibility of required medical care. Rural areas, unfortunately, were found to suffer from massive shortages of emergency care infrastructure, such as emergency care facilities and personnel, ${ }^{25}$ which suggests that there is an urgent need to establish emergency care systems providing more specialised care to rural communities.

Therefore, education and training should be provided to strengthen the paramedics' capacity, so that they can clearly identify the patient's condition and symptoms and transfer the patient to the appropriate hospital.

Additionally, it is impossible for patient to visit and select an appropriate hospital according to their symptoms. If medical staff believes that immediate treatment is not possible, it is necessary to provide the maximum first aid to ensure a safe interhospital transfer. Everyone should have guaranteed universal access to essential healthcare services regardless of region, but poor access to rural healthcare leads to health inequalities in rural areas. Opening new medical facilities in rural areas to improve healthcare access may be the best possible way to address such health inequalities, but the opening and operation of new medical facilities is never an easy task, both in terms of policy and economy, especially in rural areas lacking a full-fledged profit-making structure. Nevertheless, there is an urgent need to develop strategies for improving healthcare access in rural communities.
To this end, a healthcare policy that takes into account the regional characteristics of rural areas, rather than a government-led catch-all policy, should be formulated. Furthermore, it is necessary to establish a localised emergency care system with proper task allocation, along with a healthcare policy that constantly identifies, as well as efficiently manages and prevents health problems in rural communities, in an attempt to address interregional health inequalities.

The incompleteness of the administrative data used in this study prevented us from incorporating information on the severity of the clinical conditions of the patients at the time of visit. Such additional clinical information, if analysed in further studies, in connection with National Emergency Department Information System data, would allow the generation of more accurate results. Moreover, the distance and duration of interhospital transfer may affect the clinical outcome of patients with AMI, but actual transfer methods (ambulance, helicopter and so on), distances and times could not be determined due to the nature of the data employed in this study. If further studies process such additional information, in conjunction with the records of emergency medical dispatches and treatments from the National Fire Agency database, this would allow more detailed and accurate findings on the effects of delayed arrival times of transferred patients on clinical outcomes.

Despite the data limitations, this study still contributed to defining the basis for the risks associated with interhospital transfers and health inequalities for rural patients with AMI, by comparing the risks of interhospital transfer in patients with AMI, according to the patient's area of residence. We expect that this study will contribute to the development of sustainable healthcare policies to ensure universal healthcare services and reduce health inequalities across all regions.

\section{Author affiliations}

${ }^{1}$ Center of Biomedical Data Science, Yonsei University Wonju College of Medicine, Wonju, South Korea

${ }^{2}$ Department of Precision Medicine, Yonsei University Wonju College of Medicine, Wonju, South Korea

${ }^{3}$ Department of Occupational and Environmental Medicine, Hanyang University College of Medicine, Seongdong-gu, South Korea

${ }^{4}$ National Institute of Agricultural Science, Rural Development Administration, Jeonju, South Korea

${ }^{5}$ Department of Preventive Medicine, Yonsei University Wonju College of Medicine, Wonju, South Korea

\section{Twitter Hoon Jo @Jayson1003}

Acknowledgements We would like to thank the Korean National Health Insurance Sharing Service for providing access to the claims database (No. NHIS-2017-1-366).

Contributors HSK and SBK contributed to conception and design of the study and drafting the manuscript. HSK and DRK contributed to acquisition, analysis and interpretation of the data. IK, KL and $\mathrm{HJ}$ revised the manuscript. All of the authors approved the version to be published.

Funding This work was carried out with the support of 'Cooperative Research Program for Agriculture Science \& Technology Development (Project No.

PJ01250901)' Rural Development Administration, Republic of Korea. This work was supported by the National Research Foundation of Korea (NRF) grant funded by the Korea government (MSIT) (No. 2017R1A5A2015369). 
Competing interests None declared.

Patient consent for publication Not required.

Ethics approval This study was approved by the Institutional Review Board for Human Research Yonsei University Wonju Severance Christian Hospital (approval number: CR317313). This study analysed publicly available, anonymised and deidentified data; then, requirement for informed consents was waived.

Provenance and peer review Not commissioned; externally peer reviewed.

Data availability statement Data may be obtained from a third party and are not publicly available. Releasing of the data by the researcher is not allowed legally. All of data are available from the database of National Health Insurance Sharing Service (NHISS) https://nhiss.nhis.or.kr/bd/ab/bdaba000eng.do allows all of this data for the any researcher who promises to follow the research ethics with some cost. If you want to access the data of this article, you could download if from the website after promising to follow the research ethics.

Open access This is an open access article distributed in accordance with the Creative Commons Attribution Non Commercial (CC BY-NC 4.0) license, which permits others to distribute, remix, adapt, build upon this work non-commercially, and license their derivative works on different terms, provided the original work is properly cited, appropriate credit is given, any changes made indicated, and the use is non-commercial. See: http://creativecommons.org/licenses/by-nc/4.0/.

\section{ORCID iDs}

Hye Sim Kim http://orcid.org/0000-0002-7431-9722

Inah Kim http://orcid.org/0000-0001-9221-5831

\section{REFERENCES}

1 Health Insurance Review \& Assessment Service. Development of registration system and identification of prognostic factors for acute stroke and acute myocardial infarction. Seoul, Korea: National Emergency Medical Center, 2007.

2 Ryu JY, EK E, Kim YJ, et al. Factors associated with delayed arrival at the hospital in cases of acute stroke. J Korean Soc Emerg Med 2000;11:296-304.

3 Holland CM, Lovasik BP, Howard BM, et al. Interhospital transfer of neurosurgical patients: implications of timing on hospital course and clinical outcomes. Neurosurgery 2017;81:450-7.

4 Kahlon TS, Barn K, Akram MMA, et al. Impact of pre-hospital electrocardiograms on time to treatment and one year outcome in a rural regional ST-segment elevation myocardial infarction network. Catheter Cardiovasc Interv 2017;89:245-51.

5 Kawecki D, Gierlotka M, Morawiec B, et al. Direct admission versus interhospital transfer for primary percutaneous coronary intervention in ST-segment elevation myocardial infarction. JACC CardiovasC Interv 2017:10:438-47.

6 Mohr NM, Harland KK, Shane DM, et al. Inter-Hospital transfer is associated with increased mortality and costs in severe sepsis and septic shock: an instrumental variables approach. J Crit Care 2016;36:187-94.

7 Nacht J, Macht M, Ginde AA. Interhospital transfers from U.S. emergency departments: implications for resource utilization, patient safety, and regionalization. Acad Emerg Med 2013;20:888-93.
8 Wild J, Younus JM, Malekpour M, et al. The effect of interhospital transfers on the outcome of rural trauma. Am Surg 2017;83:39-44.

9 Lee SH, Song KJ, Shin SD, et al. The relationship between clinical outcome in subarachnoidal hemorrhage patients with emergency medical service usage and interhospital transfer. J Korean Med Sci 2015;30:1889-95.

10 Bhuyan SS, Wang Y, Opoku S, et al. Rural-Urban differences in acute myocardial infarction mortality: evidence from Nebraska. $J$ Cardiovasc Dis Res 2013;4:209-13.

11 Mohr NM, Wong TS, Faine B, et al. Discordance between patient and clinician experiences and priorities in rural interhospital transfer: a mixed methods study. J Rural Health 2016;32:25-34.

12 O'Connor RE, Nichol G, Gonzales L, et al. Emergency medical services management of ST-segment elevation myocardial infarction in the United States--a report from the American Heart Association Mission: Lifeline Program. Am J Emerg Med 2014;32:856-63.

13 Baldwin L-M, Chan L, Andrilla CHA, et al. Quality of care for myocardial infarction in rural and urban hospitals. J Rural Health 2010;26:51-7.

14 Sheikh K, Bullock C. Urban-Rural differences in the quality of care for Medicare patients with acute myocardial infarction. Arch Intern Med 2001;161:737-43.

15 Fleet R, Bussières S, Tounkara FK, et al. Rural versus urban academic hospital mortality following stroke in Canada. PLoS One 2018;13:e0191151

16 Shin JW. Regional distribution of medical expenditure and amenable mortality through treatment. Sejong, Korea: Korea Institute for Health and Social Affairs, 2014.

17 Ministry of Health and Welfare. Social work intergration manual. Sejong, Korea: Ministry of Health and Welfare, 2017.

18 Gillman L, Fatovich D, Jacobs I. Mortality of interhospital transfers originating from an emergency department in Perth, Western Australia. Australas Emerg Nurs J 2013;16:144-51.

19 Garwe T, Cowan LD, Neas BR, et al. Directness of transport of major trauma patients to a level I trauma center: a propensity-adjusted survival analysis of the impact on short-term mortality. $J$ Trauma 2011;70:1118-27.

20 Kindermann D, Mutter R, Pines JM. Emergency Department Transfers to Acute Care Facilities, 2009: Statistical Brief \#155. In: Healthcare cost and utilization project (HCUP) statistical Briefs. Rockville, MD: Agency for Healthcare Research and Quality (US), 2013.

21 Gurwitz JH, Goldberg RJ, Malmgren JA, et al. Hospital transfer of patients with acute myocardial infarction: the effects of age, race, and insurance type. Am J Med 2002;112:528-34.

22 Ellerbeck EF, Bhimaraj A, Perpich D. Organization of care for acute myocardial infarction in rural and urban hospitals in Kansas. $J$ Rural Health 2004;20:363-7.

23 Sokol-Hessner L, White AA, Davis KF, et al. Interhospital transfer patients discharged by academic hospitalists and general internists: characteristics and outcomes. J Hosp Med 2016;11:245-50.

24 Sharp SP, Ata A, Valerian BT, et al. Complications and surgical outcomes after interhospital transfer vs direct admission in colorecta surgery: a national surgical quality improvement program analysis. Am J Surg 2017;213:1031-7.

25 Song MR, SC N. The quality of life of rural residents. Seoul, Korea: Korea Rural Economic Institute, 2013. 